\section{Case Reports in Ophthalmology}

Case Rep Ophthalmol 2017;8:396-400

DOI: $10.1159 / 000478694$ Published online: July 25, 2017
(C) 2017 The Author(s)

Published by S. Karger AG, Basel www.karger.com/cop

\title{
Aggressive Posterior Retinopathy of Prematurity in a Premature Male Infant
}

\author{
Jun Zhou ${ }^{a}$ Zheng Liu ${ }^{b}$ Hai-Yan Ying ${ }^{c}$ Tao Liu ${ }^{a}$ \\ ${ }^{a}$ Department of Ophthalmology, 3201 Hospital, Xi'an Jiaotong University Health Science \\ Center, Hanzhong, China; ${ }^{b}$ Department of Pathology and Molecular Medicine, \\ 3201 Hospital, Xi'an Jiaotong University Health Science Center, Hanzhong, China; \\ 'Department of Pediatrics, 3201 Hospital, Xi'an Jiaotong University Health Science \\ Center, Hanzhong, China
}

\section{Keywords}

Retinopathy of prematurity · Premature infant · Blindness

\begin{abstract}
A premature male infant was born at 30 weeks' gestation with a birth weight of $1,700 \mathrm{~g}$ in a rural hospital. He was diagnosed with respiratory distress syndrome and received continuous positive airway pressure treatment for 26 days. At 26 days after birth, the patient was transferred to our hospital for further evaluation and management. A comprehensive eye examination revealed a stage 3 retinopathy of prematurity (ROP) involving zone 2 in both eyes. The patient was recommended to a provincial-level eye hospital for emergency laser therapy. Five months after birth, the feedback from the eye hospital showed that the patient had a high risk of blindness in both eyes. Our case report shows that delaying first screening examination increases the possibility of developing aggressive posterior ROP in infants with ROP. Doctors in rural hospitals should be aware of this possibility and trained for early screening and treatment in high-risk infants.




\section{Case Reports in Ophthalmology}

\section{Introduction}

Aggressive posterior retinopathy of prematurity (AP-ROP) is a severe and rare form of ROP which is characterized by fast progression to an advanced stage with flat neovascularization in zone 1 or zone $2[1,2]$. Previously, AP-ROP was referred to as type II ROP or rush-type ROP [3]. In the 2005 revised report of the International Committee for the Classification of Retinopathy of Prematurity, type II ROP or rush-type ROP were termed AP-ROP. This type of eye disease has the following characteristics: (1) more posterior location; (2) rapid progression, rather than through the classic stages 1-5; and (3) poor prognosis despite early treatment [4]. AP-ROP generally occurs in premature (gestational ages [GA] $<28$ weeks) and low-birth weight (BW) infants $(<1,000 \mathrm{~g})$ [5]. We report a case of AP-ROP in a premature infant treated with oxygen therapy in a rural hospital who had a late eye examination.

\section{Case Report}

A male baby was born at 30 weeks' GA, weighing 1,700 g in a rural hospital. Apgar scores were 10-10-10. He was diagnosed with respiratory distress syndrome and received continuous positive airway pressure (CPAP) treatment for 26 days. The oxygen saturation level in his blood was no higher than $40 \%$ during treatment. At 26 days after birth, the patient was transferred to our hospital. Owing to the low BW, GA, and the duration of postnatal supplemental oxygen therapy, he was at risk of developing ROP and therefore eligible for ROP screening. Figure 1 shows that his right eye had tortuosity and dilatation of temporal posterior pole vessels along with scattered hemorrhages. Severe ROP was confirmed in the left eye as well as the right eye (Fig. 2a). It was apparent on examination that both eyes exhibited stage 3 ROP in zone 2 . The left eye also showed features of "plus disease," with increased dilation of the retinal vasculature and large vascular loops as shown in Figure $2 \mathrm{~b}$. After careful examination and evaluation by ophthalmologists and retinal specialists in our hospital, a diagnosis of AP-ROP was made and the patient was recommended to a provinciallevel eye hospital for emergency laser therapy. Five months after birth, the feedback from the eye hospital showed that laser treatment was not successful and the patient had a high risk of blindness in both eyes due to treatment delay.

\section{Discussion}

A study by Fortes Filho et al. [5] showed that most cases of AP-ROP occurred in extremely premature infants with a GA $<28$ weeks and BW $<1,000 \mathrm{~g}$. Our study demonstrates that AP-ROP can occur in infants at more advanced GA and with greater BW. In a recent study from India, $15.91 \%$ of infants developing AP-ROP had a BW $>1,500 \mathrm{~g}$ with a GA $>30$ weeks [6]. Nicoară et al. [7] found that $30.43 \%$ of infants with AP-ROP had a GA $>30$ weeks and $8.70 \%$ had a BW $>1,500 \mathrm{~g}$ in Romania. In the UK, the British Association of Perinatal Medicine recommend screening of infants with a GA $<32$ weeks or a BW $<1,501$ g according to the UK screening guidelines by the Royal College of Ophthalmologists [8]. In China, the ROP screening guidelines were recommended by the Ministry of Health in 2004. Infants who meet the following criteria need to be screened: BW $\leq 2,000 \mathrm{~g}$ and/or GA $\leq 34$ weeks [9]. The guidelines include more mature infants than the UK criteria in order not to miss in- 
fants needing treatment. Unfortunately, our patient did not have an eye examination while he was hospitalized in the rural hospital. This may be due to shortage in pediatric ophthalmologists or reflect the lack of pediatric ophthalmology training in rural areas. It has been known for decades that treatment with high oxygen saturation level in the first few weeks of preterm infants may cause ROP. However, some recent studies showed that the risk of ROP was actually decreased at high levels of oxygen saturation. Thomas et al. [10] reported that maintaining oxygen saturation above $95 \%$ could decrease the incidence of ROP in premature infants (average weight at 2,508 g). Another study reported that a lower oxygen saturation level at a younger GA $(<34$ weeks) and a higher oxygen saturation level at an older GA ( $>34$ weeks) could decrease the incidence of ROP [11]. In our case, CPAP treatment with a low oxygen saturation level $(<40 \%)$ was given for 26 days and we suspect that ROP might be caused by improper oxygen therapy, but this needs to be further investigated.

In conclusion, we report an AP-ROP case due to missing ROP screening and treatment delay in a rural hospital. Our report suggests that the doctors who work in rural region should be trained in pediatric ophthalmology annually.

\section{Statement of Ethics}

This case report is in full adherence to the Declaration of Helsinki and all Federal and State laws.

\section{Disclosure Statement}

The authors have no conflicts of interest to disclose.

\section{References}

1 International Committee for the Classification of Retinopathy of Prematurity: The international classification of retinopathy of prematurity revisited. Arch Ophthalmol 2005;123:991-999.

-2 Drenser KA, Trese MT, Capone A Jr: Aggressive posterior retinopathy of prematurity. Retina 2010;30(suppl 4):S37-S40.

-3 Shah PK, Prabhu V, Karandikar SS, Ranjan R, Narendran V, Kalpana N: Retinopathy of prematurity: past, present and future. World J Clin Pediatr 2016;5:35-46.

-4 Ahn SJ, Kim JH, Kim SJ, Yu YS: Capillary-free vascularized retina in patients with aggressive posterior retinopathy of prematurity and late retinal capillary formation. Korean J Ophthalmol 2013;27:109-115.

-5 Fortes Filho JB, Eckert GU, Procianoy L, Barros CK, Procianoy RS: Incidence and risk factors for retinopathy of prematurity in very low and in extremely low birth weight infants in a unit-based approach in southern Brazil. Eye (Lond) 2009;23:25-30.

-6 Sanghi G, Dogra MR, Das P, Vinekar A, Gupta A, Dutta S: Aggressive posterior retinopathy of prematurity in Asian Indian babies: spectrum of disease and outcome after laser treatment. Retina 2009;29:13351339.

-7 Nicoară SD, Ștefănuţ AC, Nascutzy C, Zaharie GC, Toader LE, Drugan TC: Regression rates following the treatment of aggressive posterior retinopathy of prematurity with bevacizumab versus laser: 8-year retrospective analysis. Med Sci Monit 2016;22:1192-1209.

8 Wilkinson AR, Haines L, Head K, Fielder AR; Guideline Development Group of the Royal College of Paediatrics and Child Health; Royal College of Ophthalmologists; British Association of Perinatal Medicine: UK retinopathy of prematurity guideline. Eye (Lond) 2009;23:2137-2139.

-9 Xu Y, Zhou X, Zhang Q, Ji X, Zhang Q, Zhu J, et al: Screening for retinopathy of prematurity in China: a neonatal units-based prospective study. Invest Ophthalmol Vis Sci 2013;54:8229-8236. 
Case Reports in
Ophthalmology

Case Rep Ophthalmol 2017;8:396-400

DOI: $10.1159 / 000478694$

(C) 2017 The Author(s). Published by S. Karger AG, Basel www.karger.com/cop

Zhou et al.: Aggressive Posterior Retinopathy of Prematurity in a Premature Male Infant

10 Thomas WJ, Rauser M, Dovich JA, Dustin L, Flaxel CJ: Oxygen saturation in premature infants at risk for threshold retinopathy of prematurity. Eur J Ophthalmol 2011;21:189-193.

11 Sears JE, Pietz J, Sonnie C, Dolcini D, Hoppe G: A change in oxygen supplementation can decrease the incidence of retinopathy of prematurity. Ophthalmology 2009;116:513-518.

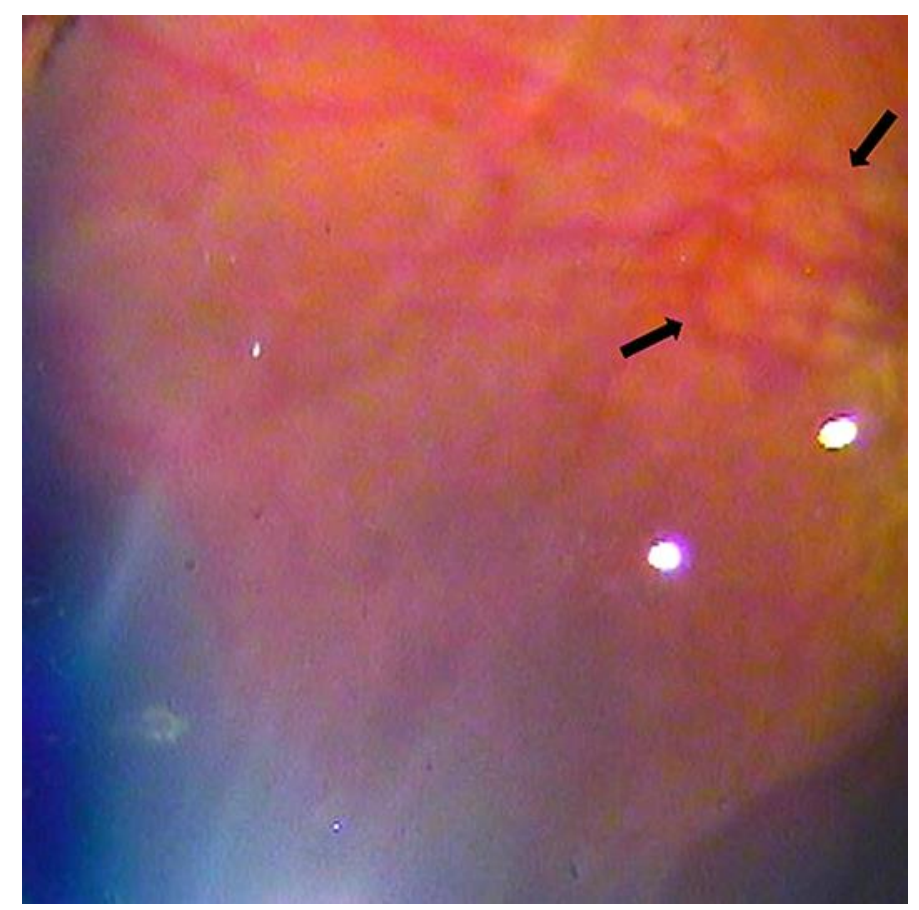

Fig. 1. Fundus photograph of stage $3 \mathrm{ROP}$ in the right eye. The temporal posterior pole vessels show tortuosity and dilatation (arrows) and scattered hemorrhage throughout retina. 


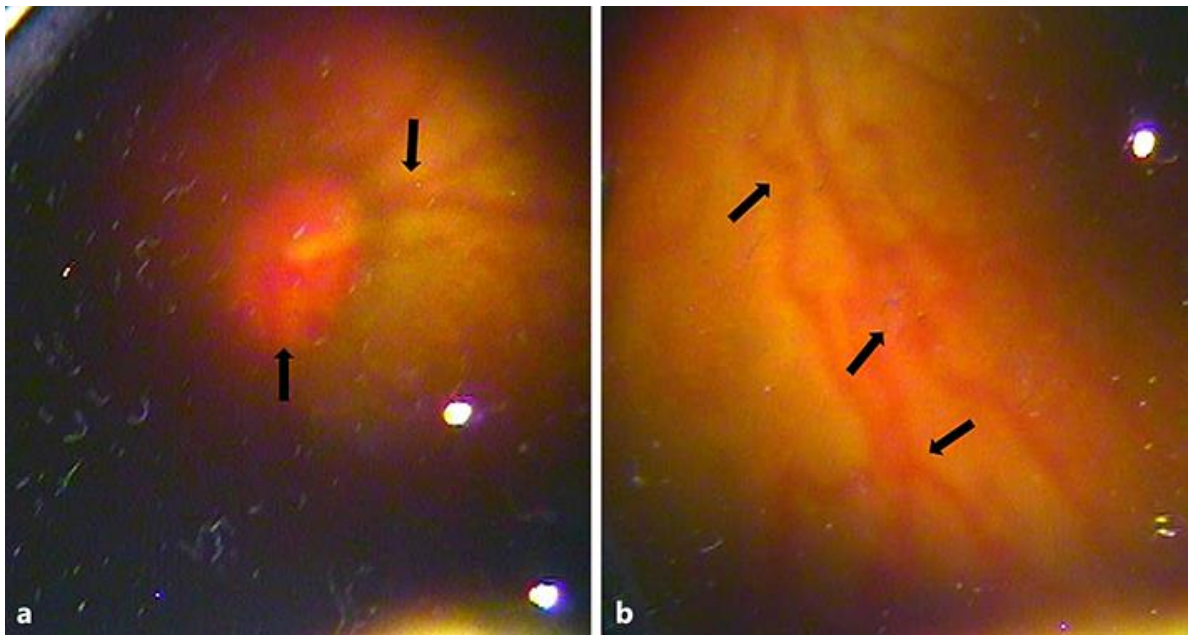

Fig. 2. Fundus photograph of stage 3 ROP and large vascular loops in left eye. a There are abnormal dilatation and tortuosity of posterior pole vessels (arrows). b Several linear hemorrhages and vascular loops are visible (arrows). 